\title{
Hybrid Vlasov simulations for alpha particles heating in the solar wind
}

\author{
Denise Perrone $^{1}$, Francesco Valentini ${ }^{2,1}$ and Pierluigi Veltri ${ }^{1}$ \\ ${ }^{1}$ Università della Calabria, Dipartimento di Fisica and CNISM, \\ Unità di Cosenza, I-87030 Arcavacata di Rende, Italy \\ email: denise.perrone@fis.unical.it; pierluigi.veltri@fis.unical.it \\ ${ }^{2}$ Università di Pisa, Dipartimento di Fisica and CNISM, 56127 Pisa, Italy \\ email: francesco.valentini@fis.unical.it
}

\begin{abstract}
Heating and acceleration of heavy ions in the solar wind and corona represent a long-standing theoretical problem in space physics and are distinct experimental signatures of kinetic processes occurring in collisionless plasmas. To address this problem, we propose the use of a low-noise hybrid-Vlasov code in four dimensional phase space (1D in physical space and $3 \mathrm{D}$ in velocity space) configuration. We trigger a turbulent cascade injecting the energy at large wavelengths and analyze the role of kinetic effects along the development of the energy spectra. Following the evolution of both proton and $\alpha$ distribution functions shows that both the ion species significantly depart from the maxwellian equilibrium, with the appearance of beams of accelerated particles in the direction parallel to the background magnetic field.
\end{abstract}

Keywords. plasmas, turbulence, waves.

Helium is the second most abundant element in the Sun and plays an important and not yet understood role in the dynamics of the solar wind, corona and interior. In situ measurements in the solar wind have clearly shown that the heavy minor ions in this essentially collisionless space plasma are heated and accelerated preferentially as compared to protons and electrons. These observations indicate that there are sources of ion heating and momentum exchange which operate differently on protons and $\alpha$ throughout solar wind (Kasper et al., 2008, Hansteen et al., 1997). The investigation of these preferential effects has pushed several workers to study the quasi-linear resonant cyclotron interaction of solar wind ions with parallel-propagating ion cyclotron waves (Dusenbery \& Hollweg, 1981; Marsch et al., 1982, Isenberg \& Hollweg, 1983; Isenberg, 1984; Hollweg \& Isenberg, 2002; Xie \& Ofman, 2004; Ofman, 2010).

The fast technological development of supercomputers gives the possibility of using kinetic Eulerian Vlasov codes that solve the Vlasov-Maxwell equations in multidimensional phase space. The use of these "zero-noise" codes is crucial since Eulerian algorithms (Mangeney et al., 2002, Valentini et al., 2007) allow for the first time the analysis of kinetic effects in the small-scale tail of the turbulent cascade, where the energy level of the fluctuations is typically very low. In this spectral region, Lagrangian PIC algorithms fail due to their intrinsic noise. In recent years, thank to the use of this code (4D or $5 \mathrm{D})$, significant steps forward are made in the analysis of the evolution of solar wind turbulence towards dissipation (Valentini et al., 2008; Valentini \& Veltri, 2009; Valentini et al., 2010; Valentini et al., 2010). These numerical results have shown that, at least for $T_{e} / T_{p}>3-4$, newly identified electrostatic (acoustic-like) modes, longitudinal with respect to the average magnetic field and driven by particle distribution functions far from local thermodynamic equilibrium, represent a privileged channel for the turbulence to carry the energy towards small disordered scales. 

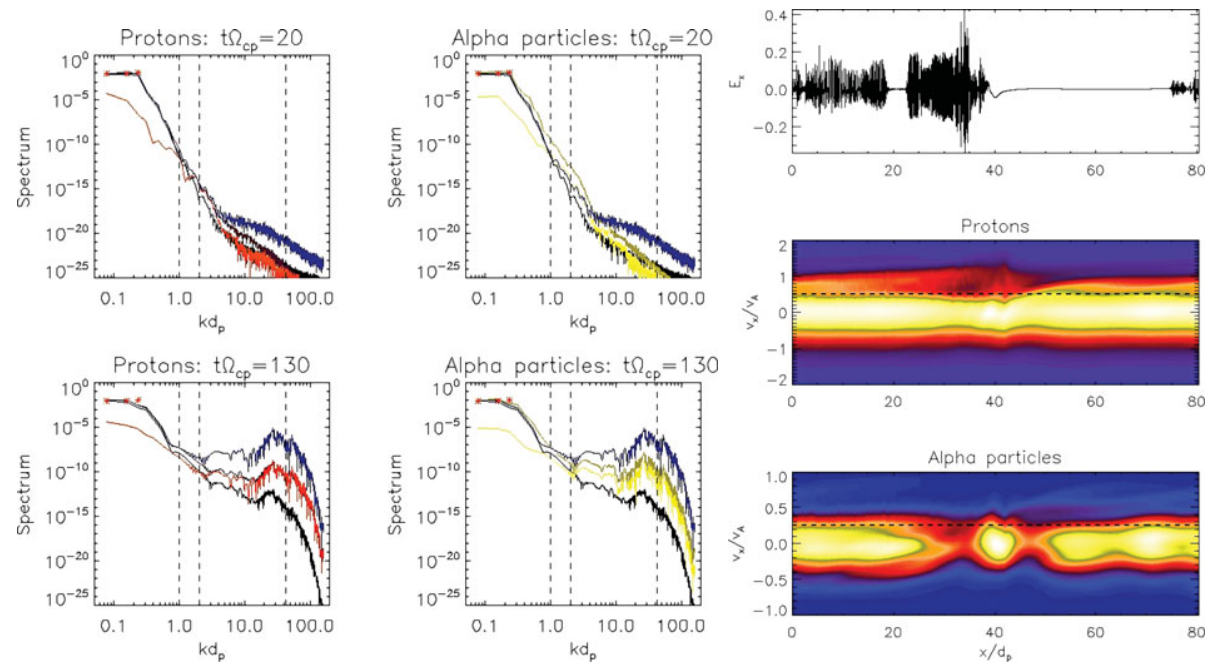

Figure 1. Energy spectra at two different times: magnetic (black line) and electric energy (blue line). For protons (left): kinetic energy (purple line) and density (red line). For $\alpha$ particles (middle): kinetic energy (yellow line) and density (green line). (Right) Parallel electric field versus $x$ at $t=150$ (top plot). $x-v_{x}$ level lines of the reduced distribution for protons (middle plot) and for $\alpha$ (bottom plot) at $t=150$.

In the present paper, we propose the use of an updated version of the hybrid-Vlasov code, which includes the kinetic dynamics of heavy ions. Electrons are treated as a fluid and a generalized Ohm equation, that retains Hall effect and electron inertia terms, is considered. Faraday equation, Ampère equation (in which the displacement current is neglected) and an equation of state for the electron pressure close the system. Quasineutrality is assumed. The solutions of the above equations are obtained through a numerical hybrid-Vlasov code in 4D (1D in space and 3D in velocity) phase space. Periodic boundary conditions are imposed in physical space. Times are scaled by the proton cyclotron frequency $\Omega_{c p}$, lengths by the proton skin depth $d_{p}=v_{A} / \Omega_{c p}, v_{A}$ being the Alfvén speed, and masses by the proton mass $m_{p}$. We simulate a plasma embedded in a background magnetic field $\mathbf{B}=B_{0} \mathbf{e}_{x}, x$ being the direction of wave propagation. The system is perturbed at $t=0$ by a circularly left hand polarized Alfvén wave in the perpendicular plane. We choose an isothermal equation of state for the electron pressure $P_{e}=(\beta / 2) n_{e} T_{e} / T_{p}$, with $\beta=2 v_{t p}^{2} / v_{A}^{2}=0.5, T_{e} / T_{p}=10$ and $T_{\alpha}=T_{p}$. The mass ratios are $m_{e} / m_{p}=1 / 1836$ and $m_{\alpha} / m_{p}=4$, the charge number is $Z_{\alpha}=2$ and the density ratio is $n_{\alpha}^{(0)} / n_{p}^{(0)}=5 \%$. The length of the physical domain is $L_{x}=12.8 d_{p}$, while the limits of the velocity domain in each direction are fixed at $v_{i}^{\max }=5 v_{t h_{i}}$. We use 4096 gridpoints in physical space and $51^{3}$ in velocity space. At $t=0$, the first three modes in the spectrum of velocity and magnetic perturbations are excited with amplitude $\epsilon=0.5$. No density disturbance is imposed at $t=0$. The simulation is carried up to $t=200$.

Figure 1 (left and middle) reports the numerical spectra of particle density $\left(\left|n_{k}^{(p)}\right|^{2}\right.$, red line, $\left|n_{k}^{(\alpha)}\right|^{2}$, green line), kinetic $\left(\left|U_{k}^{(p)}\right|^{2}\right.$, purple line; $\left|U_{k}^{(\alpha)}\right|^{2}$, yellow line), magnetic $\left(\left|B_{k}\right|^{2}\right.$, black line) and electric $\left(\left|E_{k}\right|^{2}\right.$, blue line) energies. The four plot refer to two different times in the simulations for protons and $\alpha$ particles. The same phenomenology described for the protons by Valentini et al., 2008, is observed: the energy transfer to small scales is not driven by a continuos cascade, but for $t>60$ a well defined group of wavenumbers are excited. The energy spectra are evidently peaked around $k \simeq 30$ and the magnetic energy is sensibly lower than electric one, meaning that electrostatic 

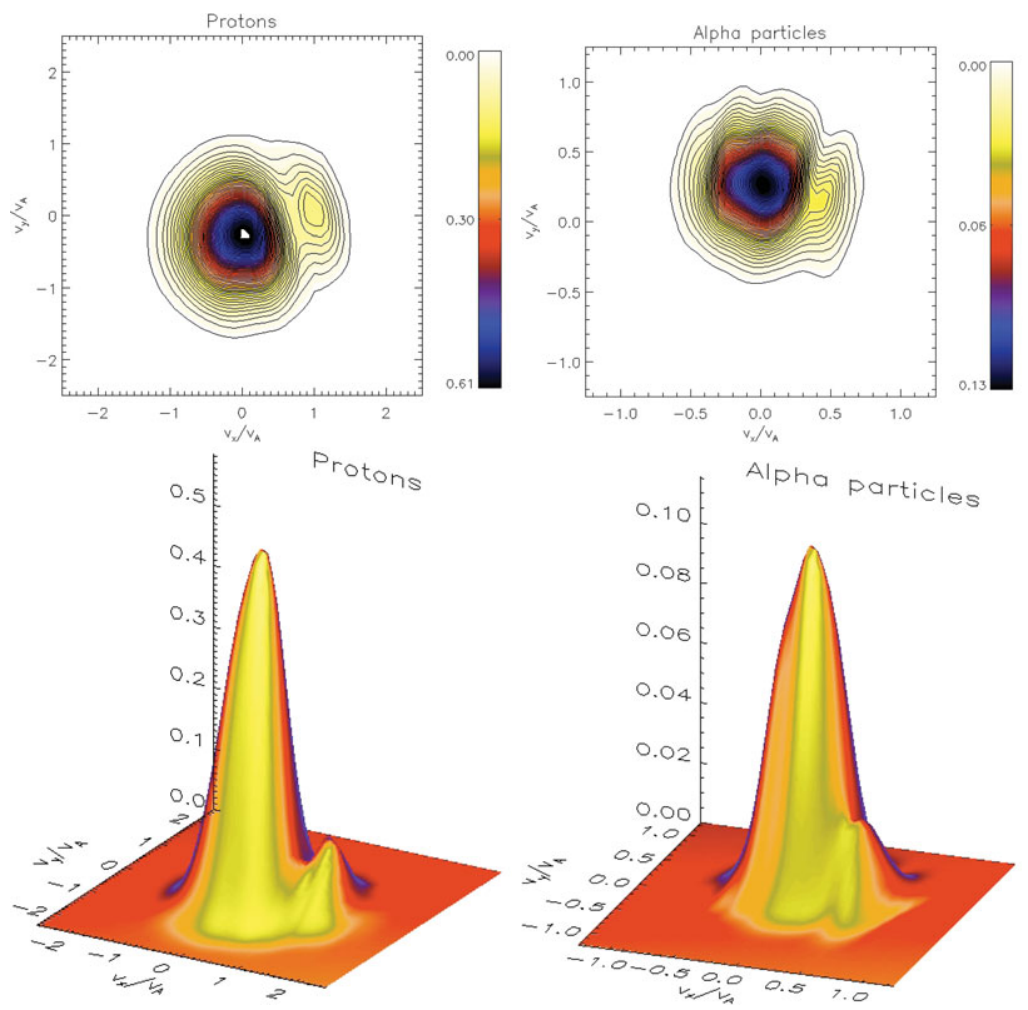

Figure 2. Level lines (at the top) and surface plot (at the bottom) of protons (left) and $\alpha$ particles (right) distributions in the velocity plane $v_{x}-v_{y}$ at $t=150$.

activity is observed. The top right plot of Fig. 1 shows $E_{x}$ as a function of $x$ at $t=150$; the middle and the bottom plots show the $x-v_{x}$ level lines of the reduced distribution for protons and $\alpha$ respectively. Phase space holes are generated in correspondence of the regions of highly impulsive behavior of $E_{x}$. These vortices are the typical signature of particles trapping. Another important phenomenon, recovered in correspondence of the maximum intensity of electrostatic activity in solar wind, is the generation of a doublestream ion distribution. These secondary beams, that move along the ambient field with different velocities, are shown in Fig. 2. The top plot displays the $v_{x}-v_{y}$ contour lines of $f$ at $t=150$ integrated over $v_{z}$ and for a given point $x_{M}$ in the physical domain, with $x_{M}$ being the point where the $x-v_{x}$ trapped region has maximum velocity width. The bottom To identify the short-wavelength fluctuations observed in the simulations, we considered the $\kappa-\omega$ spectrum of the parallel electric field energy. This Fourier analysis displays the presence of only one branch of electrostatic waves, driven by kinetic trapping effects. The ion-acoustic waves, discussed in Valentini \& Veltri (2009), are not present. These simulations show that the gross features of the kinetic effects occurring across the ion skin depth are not affected by the presence of $\alpha$ particles. Nevertheless this introduction allows to study the evolution of their distribution function and gives rise to some peculiar behavior.

\section{References}

Dusenbery, P. B. \& Hollweg, J. V. 1981, J. Geophys. Res. 86, 153

Hansteen, V. H., Leer, E., \& Holzer, T. E. 1997, ApJ 482, 498 
Hollweg, J. V. \& Isenberg, P. A. 2002, J. Geophys. Res. 107, A7, 1147

Isenberg, P. A. \& Hollweg, J. V. 1983, J. Geophys. Res. 88, 3924

Isenberg, P. A. 1984, J. Geophys. Res. 89, A4, 2133

Kasper, J. C., Lazarus, A. J., \& Gary, S. P. 2008, Phys. Rev. Lett. 101, 261103

Mangeney, A., Califano, F., Cavazzoni, C., \& Travnicek, P. 2002, J. Comput. Phys. 179, 405

Marsch, E., Goertz, C. K., \& Richter, K. 1982a, J. Geophys. Res. 87, 5030

Ofman, L. 2010, J. Geophys. Res. 115, A04108

Valentini, F., Trávníček, P., Califano, F., Hellinger, P., \& Mangeney, A. 2007 J. Comput. Phys. 225,753

Valentini, F., Veltri, P., Califano, F., \& Mangeney, A. 2008, Phys. Rev. Lett. 101, 025006

Valentini, F. \& Veltri, P. 2009, Phys. Rev. Lett. 102, 225001

Valentini, F., Califano, F., \& Veltri, P. 2010, Planetary and Space Science doi:10.1016/j.pss.2009.11.007 (in press)

Valentini, F., Califano, F., \& Veltri, P. 2010, Phys. Rev. Lett. 104, 205002

Xie, H. \& Ofman, L. 2004, J. Geophys. Res. 109, A08103 